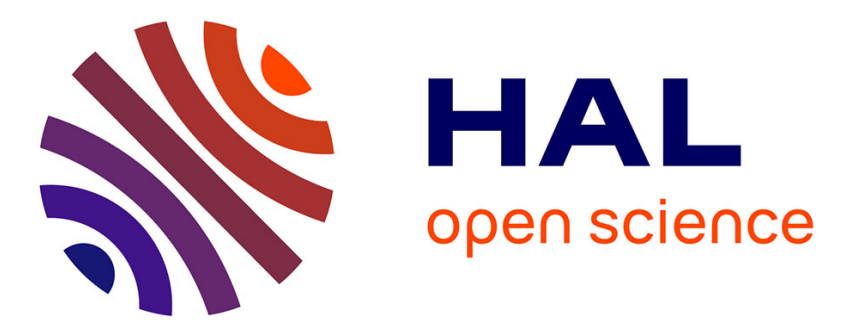

\title{
SIMULATION DES EFFETS DES IMPACTS À HYPERVITESSE
}

\author{
J. Leyrat, E. Charvet, M. Lacomme, P. Massard, H. Pujols
}

\section{To cite this version:}

J. Leyrat, E. Charvet, M. Lacomme, P. Massard, H. Pujols. SIMULATION DES EFFETS DES

IMPACTS À HYPERVITESSE. Journal de Physique IV Proceedings, 1991, 01 (C3), pp.C3-155-C3162. 10.1051/jp4:1991320 . jpa-00250462

\section{HAL Id: jpa-00250462 https://hal.science/jpa-00250462}

Submitted on 1 Jan 1991

HAL is a multi-disciplinary open access archive for the deposit and dissemination of scientific research documents, whether they are published or not. The documents may come from teaching and research institutions in France or abroad, or from public or private research centers.
L'archive ouverte pluridisciplinaire HAL, est destinée au dépôt et à la diffusion de documents scientifiques de niveau recherche, publiés ou non, émanant des établissements d'enseignement et de recherche français ou étrangers, des laboratoires publics ou privés. 


\title{
SIMULATION DES EFFETS DES IMPACTS À HYPERVITESSE
}

\author{
J.P. LEYRAT*
H.C. PUJOLS \\ * Centre d'Etudes de Limeil-Valenton, F-94195 \\ Villeneuve-Saint-Georges cedex, France \\ * CEA-CESTA, F-94195 Villeneuve-Saint-Georges cedex, France
}

\begin{abstract}
Résumé - Pour étudier les effets des impacts à hypervitesse, nous avons mis au point un propulseur qui utilise l'onde de détonation engendrée dans un cylindre d'explosif pour projeter des masses compactes de l'ordre du gramme à des vitesses allant de 5 a $10 \mathrm{~km} / \mathrm{s}$. Les cibles testées sont des disques en alliage d'aluminium ou de titane, dont l'épaisseur varie de 15 à $50 \mathrm{~mm}$.. Les simulations numériques ont été effectuées avec le code $2 \mathrm{D}$ aux différences finies CEL; ce code permet d'utiliser diverses équations d'état (équations SESAME, équations WILKINS...), ainsi qu'une loi de comportement dynamique (modèle de STEINBERG-COCHRAN-GUINAN). La comparaison expériences-calculs est trés bonne en ce qui concerne les dimensions du cratère formé, l'épaisseur critique de cible pour laquelle on observe un début d'écaillage, ainsi que l'épaisseur de l'écaille.
\end{abstract}

\begin{abstract}
An experimental study of hypervelocity impact was performed with a propeller using the detonation wave of an explosive cylinder to project compact masses of about 1 gram at 5 to $10 \mathrm{~km} / \mathrm{s}$. The targets were large disks of aluminum or titanium alloys of different thicknesses $(15$ to $50 \mathrm{~mm})$. For the numerical correlation, we used the 2D finite differences code CEL, with accurate equations of state (either SESAME or WILKINS E.O.S.) and a dynamic behaviour law (the STEINBERG-COCHRAN-GUINAN model). Under these conditions, the code correlates well the depth and diameter of the crater, the critical dimensions of the target for which spalling is observed, and the thickness of the spall.
\end{abstract}

\section{Introduction}

Les effets des impacts sur des cibles de différentes natures (métalliques, composites à structure tissée, multicouches...) dépendent beaucoup de la vitesse du projectile. Dans le domaine des impacts à hypervitesse, c'est-à-dire quand la vitesse du projectile est supérieure à la vitesse du son dans les divers matériaux (typiquement au delà de $5 \mathrm{~km} / \mathrm{s}$ pour des métaux "classiques" tẹs l'aluminium, le titane ou le fer), les đéformations sont suffisamment rapides pour que l'échauffement adiabatique fonde ou vaporise une partie, voire la totalité du projectile, ainsi que la cible aux environs du point d'impact. Dans ce cas, l'explósion de la zone vaporisé gouverne le choc qui se propage dans la cible, et, par conséquent, les endommagements qui s'ensuivent.

Rappelons que, qualitativement, les impacts à hypervitesse se caractérisent par trois types d'effets (figure 1) :

- à faible vitesse d'impact, ou pour des cibles épaisses, un cratère est formé, caractérisé par son diamètre et sa profondeur ;

- à plus grande vitesse, et/ou pour des cibles plus minces, une écaille est arrachée en face arrière du projectile; elle est définie par son diamètre et par son épaisseur, à peu prés constante ;

- à trés grande vitesse, et/ou pour des cibles encore plus minces, on obtient une perforation complète de la cible.

Pour étudier ces phénomènes hautement dynamiques, il est nécessaire de disposer de codes de calculs performants, munis de lois de comportement validées par de nombreuses expériences.Nous présentons dans cette étude les moyens expérimentaux et numériques actuellement à notre disposition pour traiter ce problème, ainsi que les résultats des premières comparaisons experiences-calculs. 

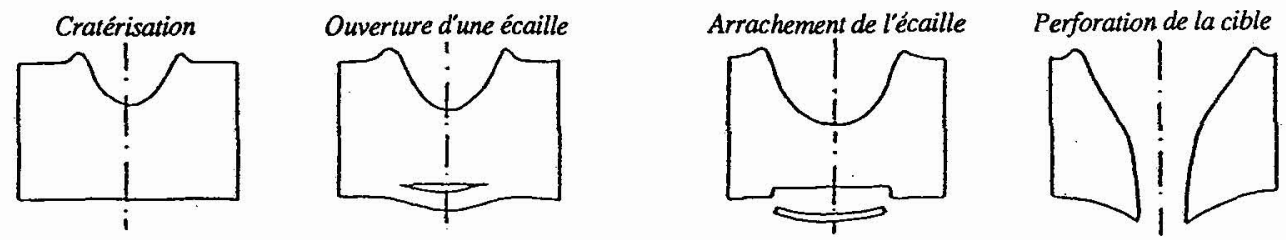

Figure 1-Effets des impacts à hypervitesse

\section{Les expériences}

\section{Le dispositif expérimental}

Le propulseur mis au point pour réaliser des expériences d'impact à grande vitesse comporte un générateur à explosif dont la đétonation met en mouvement un ensemble composé d'un petit cylindre central (le projectile proprement dit) et d'une couronne périphérique. Le cylindre se détache progressivement de la couronne, freinée par les détentes latérales, et qui se fragmente par élongation. Un pare-éclats permet de dévier les débris hors de l'axe du montage, de manière à ce que seul le cylindre impacte la cible (figure 2 - a). Une variante du montage permet de se dispenser de la présence du pare-éclats, en utilisant une couronne "conique" (figure 2 - b). Un système de radiographie X permet de visualiser la forme du projectile juste avant l'impact, et de vérifier s'il attaque la cible perpendiculairement à son axe.

Avec ce lanceur monoétage, il est possible de projeter des masses de quelques centaines de milligrammes à quelques grammes à des vitesses n'excédant pas $7 \mathrm{~km} / \mathrm{s}$. Pour améliorer ces performances, on peut utiliser un propulseur multi-étages (figure 3), où la détonation de l'explosif de l'étage d'ordre $\mathbf{n}$ est initiée par l'impact du projectile d'ordre $\mathbf{n}-1$. A condition que la masse surfacique de chaque étage soit inférieure à celle du projectile précédent, on peut ainsi dépasser $10 \mathrm{~km} / \mathrm{s} / 1 /$.

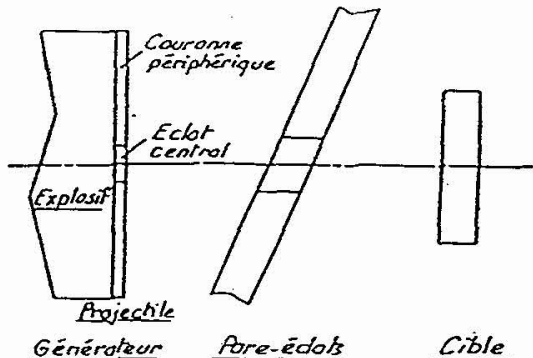

a - avec pare-éclats

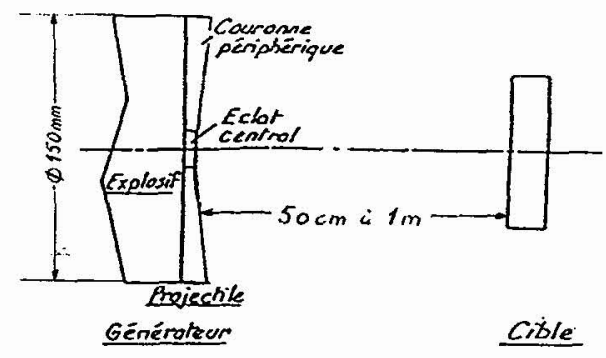

b - sans pare-eclats

Figure 2 - Principe du générateur monoétage

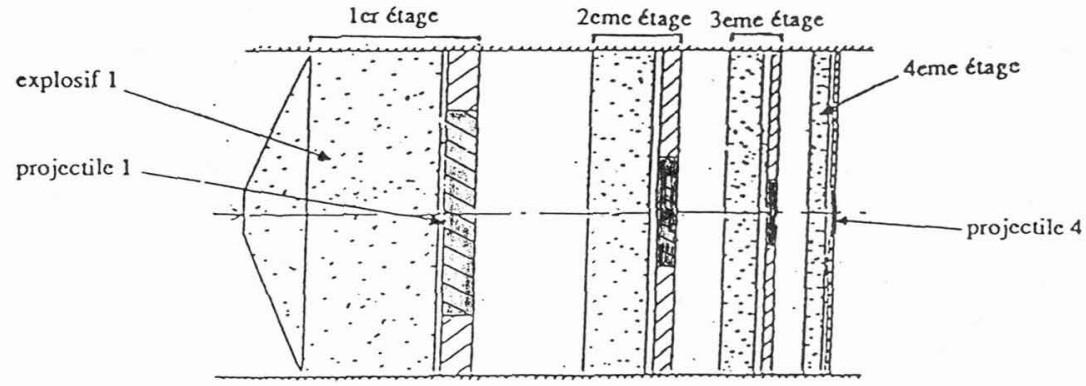

Figure 3 - Principe d'un propulseur multiétages 


\section{Les résultats expérimentaux}

Les premières campagnes de tirs avaient essentiellement pour but de valider le moyen d'essai et d'explorer les possibilités des codes numériques. Trois configurations d'impact ont été étudiées :

- Impact $A U 4 G \rightarrow A U 4 G$ à $5400 \mathrm{~m} / \mathrm{s}$ : dans ce cas, le projectile, en alliage d'aluminium AU4G, est un cylindre de diamètre $12 \mathrm{~mm}$ et de hauteur $6,8 \mathrm{~mm}$; sa masse est de 2,15 grammes, et son énergie cinétique au moment de l'impact est de $31,35 \mathrm{~kJ}$. Les cibles, également en $A U 4 \mathrm{G}$, sont des disques de grand diamètre (200 $\mathrm{mm}$, ce qui permet de négliger les effets de bord) et d'êpaisseurs comprises entre 35 et $50 \mathrm{~mm}$;

- Impact $A U 4 G \rightarrow$ TA6V à $5400 \mathrm{~m} / \mathrm{S}:$ avec un projectile similaire et la même vitesse d'impact, des expériences ont été réalisées avec des cibles en alliage de titane TA6V d'épaisseur 35 et $45 \mathrm{~mm}$;

- Impact AU4G $\rightarrow$ AU4G à $7200 \mathrm{~m} / \mathrm{s}$ : ces expériences d'impact à plus grande vitesse sur cibles d'AU4G minces (épaisseur 15 à $25 \mathrm{~mm}$ ) ont été effectuées avec des projectiles plus petits (diamètre $6 \mathrm{~mm}$, hauteur $2 \mathrm{~mm}$, masse 0,16 gramme) ; l'énergie cinétique au moment de l'impact est donc plus faible $(4,15 \mathrm{~kJ})$.

Les principaux résultats sont rassemblés sur le tableau 1. Ils permettent, pour les trois types d'expériences, de déterminer les épaisseurs "critiques" de cibles correspondant aux seuils d'écaillage et de perforation complète.

Tableau 1 - Principaux résuluats expérimentaux

\begin{tabular}{|c|c|c|c|c|}
\hline $\begin{array}{c}\text { type } \\
\text { d'expérience }\end{array}$ & $\begin{array}{c}\text { épaisseur } \\
\text { de cible } \\
\text { mm }\end{array}$ & $\begin{array}{c}\text { diametre } \\
\mathrm{mm}\end{array}$ & $\begin{array}{l}\text { profondeur } \\
\mathrm{mm}\end{array}$ & $\begin{array}{l}\text { épaisseur } \\
\text { d'écaille } \\
\mathrm{mm}\end{array}$ \\
\hline $\begin{array}{c}\text { AU4G/AU4G } \\
2,15 \mathrm{~g} \\
5400 \mathrm{~m} / \mathrm{s}\end{array}$ & $\begin{array}{l}50 \\
45 \\
40 \\
35\end{array}$ & $\begin{array}{l}42 \\
41 \\
42 \\
42\end{array}$ & $\begin{array}{r}20,5 \\
17,5 \\
=19 \\
\text { Perf. complète }\end{array}$ & $\begin{array}{l}\text { début d'ouverture } \\
\begin{array}{c}3 \text { a } 4 \\
=5 \\
=5\end{array}\end{array}$ \\
\hline $\begin{array}{c}\text { AU4G/TA6V } \\
2,15 \mathrm{~g} \\
5400 \mathrm{~m} / \mathrm{s}\end{array}$ & $\begin{array}{l}45 \\
35\end{array}$ & $\begin{array}{l}37 \\
37\end{array}$ & $\begin{array}{l}12 \\
12\end{array}$ & $\begin{array}{c}\text { début d'ouverture } \\
2 \text { à } 4 \\
\text { (seuil d'arrachement) }\end{array}$ \\
\hline $\begin{array}{c}\text { AU4G/AU4G } \\
0,16 \mathrm{~g} \\
7200 \mathrm{~m} / \mathrm{s}\end{array}$ & $\begin{array}{l}25 \\
20 \\
15\end{array}$ & $\begin{array}{c}17 \\
17 \\
?\end{array}$ & $\begin{array}{c}10 \\
9 \\
\text { Perf. complète }\end{array}$ & $\begin{array}{c}\text { début d'ouverture } \\
\qquad \begin{array}{c} \\
\approx 2\end{array}\end{array}$ \\
\hline
\end{tabular}

La figure 4 représente des coupes aprés tir de cibles d'AU4G impactées à $5400 \mathrm{~m} / \mathrm{s}$ par un projectile de $2,15 \mathrm{~g}$; dans le cas d'une cible épaisse $(45 \mathrm{~mm})$, on peut ainsi visualiser la forme et la profondeur du cratère, l'épaisseur d'écaille, ainsi que l'amorce de lignes de fractures qui conduisent, pour des cibles plus minces ( $35 \mathrm{~mm}$ ), à la perforation.

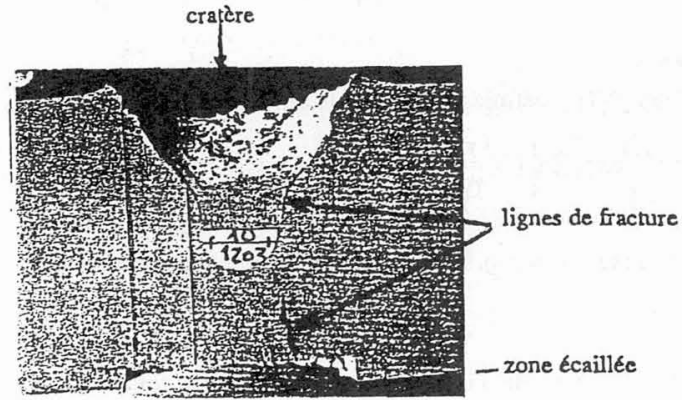

Epaisseur de cible : $45 \mathrm{~mm}$ cratérisation et arrachement d'écaille

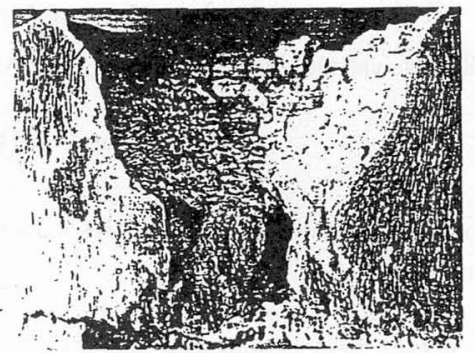

Epaisseur de cible : $35 \mathrm{~mm}$ perforation complete

Eigure 4

Coupe de cibles en $A U 4 G$ aprés impact par un projectile de $2.15 \mathrm{~g}$ a $5.4 \mathrm{~km} / \mathrm{s}$ 


\section{Les simulations numériques}

\section{Le Code. CEL}

Pour restituèr numériquèment ces expériences, nous utilisons le code bidimensionnel aux différences finies CEL. Ce code couplé Euler-Lagrange possède un schéma eulérien d'ordre 2, et permet lintroduction de lignes de glissement aux interfaces Euler-Lagrange ou Lagrange-Lagrange. De plus, on peut simuler l'ouverture d'une écaille, à l'aide d'un critère de tension maximale.

Nous avons utilisé différentes équations d'état pour ces calculs. Citons en particulier les équations SESAME de LoS Alamos (particulièrement intéressantes pour traiter les changements de phase), et l'equation de Wilkins à deux phases, dont la formulation est de type Mie-Grüneisen en compression et Puff en tension :

$$
\begin{gathered}
\rho>\rho_{0}: P=K \mu+D \mu^{2}+S \mu^{3}+\Gamma \rho E \\
\rho<\rho_{0}: P=\rho[H+(\Gamma-H) \sqrt{\eta}]\left\{E-E_{s}\left\{1-\exp \frac{K}{\Gamma \rho E_{s}} \frac{1}{\eta}\left(1 \frac{1}{\eta}\right)\right]\right\}
\end{gathered}
$$

avec : $\quad-P=$ pression hydrostatique ;

$-\rho=$ densité ;

$-\mathrm{E}=$ énergie ;

$-\mu=\rho / \rho_{0}-1, \eta=\rho / \rho_{0}$;

$-\Gamma=$ coefficient de Grüneisen ;

- $\mathrm{K}=$ module de compressibilité ;

- D, $S$ : coefficients quadratique et cubique de $\mu$ dans la formulation de $P$;

$-H=\gamma-1$, avec $\gamma=$ coefficient polytropique des gaz;

$-E_{S}=$ enthalpie de vaporisation.

Cette équation d'état est complétée par une loi de comportement élastoplastique. En effet, il n'est pas possible de simuler correctement les effets dans la cible en supposant qu'elle a un comportement purement hydrodynamique. L'apparition d'un échauffement intense au voisinage de limpact, ainsi que la diminution rapide au cours du temps de la vitesse du projectile nous conduisent à adopter pour la cible uñè loi de comportement dépendant de la température et de la vitesse de déformation. Il s'agit du modèle de STENNBERG-COCHRAN-GUINAN (ou modèle SCG) /2. Rappelons que ce modèle, mis au point pour des métaux, permet de tenir compte des variations du module de cisaillement $G$ et de la contrainte d'écoulement $Y$ du matériau avec la température $T$, la pression hydrostatique $P$, et la déformation plastique équivalente $\varepsilon_{p}$. Il est décrit par :

$$
\begin{gathered}
G=G_{P E}\left(\frac{p}{\eta^{1 / 3}}\right)+G_{0}\left[G_{P E}(T-300)+1\right] \\
Y=\left(\beta_{1}+\beta \varepsilon_{p}\right)\left[Y_{d}\left(1+G_{P T E}(T-300)\right)+Y_{P E}\left(\frac{p}{\eta^{1 / 3}}\right)\right]
\end{gathered}
$$

$$
\text { avec: } \quad\left(\beta_{1}+\beta \varepsilon_{p}\right) \leq Y_{\max }
$$

Ce modèle reste valable jusqu'à la température de fusion "vraie" $T_{\mathrm{fm}}$ définie par une loi de type Lindemann :

$$
T_{f m}=T_{f u} \eta^{2\left(\gamma_{0}-a-1 / 3\right)} \exp \left[2 a\left(1-\frac{1}{\eta}\right)\right]
$$

où $\mathrm{T}_{\mathrm{fu}}$ est la température de fusion à la pression ambiante (lorsque $\rho=\rho_{0}$ ). Pour des températures supérieures, le calcul se fait en hydrodynamique.

Au total, le traitement d'un matériau à l'aide du modèle SCG nécessite 11 paramètres en plus de la température de fusion. Certains d'entre eux ont une signification physique évidente : ainsi, $G_{0}$ et $Y_{0}$ peuvent être assimilés (en première approximation) au module de cisaillement et à la limite élastique en régime statique et à température ordinaire. La plupart des autres paramètres sont semi-empiriques, et, pour l'AU4G et le TA6V, ont été "calés" à partir d'expériences de type Taylor /3-4-5/.

A titre d'exemple, la figure 5 met en évidence l'influence de la loi de comportement dans le cas de l'impact à $5400 \mathrm{~m} / \mathrm{s}$ d'un 
projectile de $2,15 \mathrm{~g}$ sur une cible d'AU4G de $50 \mathrm{~mm}$ d'épaisseur. On constate que l'application du modèle SCG à la cible permet de retrouver la profondeur du cratere "expérimental" $(20,5 \mathrm{~mm})$, alors qu'un calcul purement hydrodynamique la surévalue nettement ; avec un modèle élastique-parfaitement plastique indépendant de la température et de la vitesse de déformation, la profondeur calculée est aus contraire trop faible.

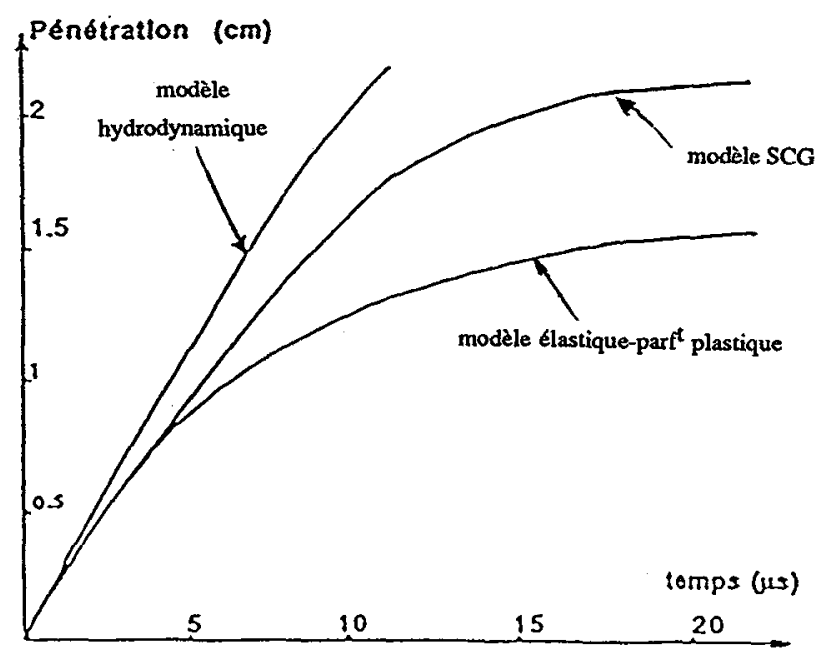

Figure 5 - Profondeur de pénétration en fonction du temps - Cible en AU4G (e=50 mm) - Projectile de $2.15 \mathrm{~g}-\mathrm{v}=5400 \mathrm{~m} / \mathrm{s}$

\section{Résultats des calculs et conclusion}

Pour visualiser la cinématique du phénomène, nous avons simulé par un calcul purement eulérien limpact à $5400 \mathrm{~m} / \mathrm{s}$ d'un projectile de $2,15 \mathrm{~g}$ sur une cible d'AU4G de $50 \mathrm{~mm}$ d'épaisseur. Le maillage comporte 8000 cellules ; le calcul, poursuivi jusqu'à $20 \mu$ s (soit un temps supérieur à un aller-retour d'onde dans l'épaisseur de la cible), dure 53 minutes sur CRAY XMP.

Sur la figure 6, on a représenté la carte de pression hydrostatique dans la cible à differents instants. L'onde de compression initialement sphérique s'amortit rapidement : de l'ordre du mégabar avant $1 \mu$ s, elle ne vaut plus que 40 kilobars vers $7 \mu$ s. Elle est suivie de compressions secondaires, plus faibles, et qui correspondent aux aller-retours d'ondes dans le projectile.

A partir de $8 \mu \mathrm{s}$, l'onde, qui continue à se propager en quasi-spherique vers la périphérie de la plaque, se réfléchit en tension sur laxe. Ces tensions atteignent un niveau important au voisinage de la surface libre : ainsi, à $9 \mu \mathrm{s}$, elles sont d'environ 19 kilobars à $3 \mathrm{~mm}$ de cette surface ; cette valeur est légèrement supérieure à la tension critique d'écaillage de l'AU4G mesurée en monodimensionnel grâce à des expériences de projection de plaques $(\approx 18 \mathrm{kbars}) 7 \%$. Ceci est cohérent avec les observations expérimentales (ouverture d'une écaille sans arrachement).

Si nous poursuivons le calcul sans écaillage, on constate un amortissement progressif de l'onde de tension au fur et à mesure de sa progression vers la face impactée, où elle se réfléchit vers $14 \mu \mathrm{s}$, tandis que l'onde de compression "primaire" atteint les bords latéraux de la cible. Les niveaux de contraintes sont alors beaucoup plus faibles (moins de 10 kilobars) et s'amortissent de plus en plus ; à $20 \mu \mathrm{s}$, la vitesse de l'interface projectile-cible est pratiquement nulle, et on peut considérer que le phénomène est terminé. La forme du cratère à cet instant est trés comparable à celle observée dans l'expérience : même profondeur de pénétration $(20,4 \mathrm{~mm}$ ), diamètre de cratère peu différent ( $40 \mathrm{~mm}$ au lieu de 42 ), forme similaire des lèvres du cratère.

Nous avons effectué un second calcul de simulation en maillant la moitié de la cible, du côté non agressé, en coordonnées de Lagrange, les mailles ayant les mêmes dimensions que les cellules eulériennes utilisées précédemment dans cette zone. Ceci présente un double intérêt :

- d'une part, le temps de calcul diminue notablement ( 35 minutes CRAY au lieu de 53 pour atteindre $20 \mu s$ ); 
- d'autre part, nous pouvons introduire un critère de tension limite pour traiter l'ouverture de l'écaille. En prenant comme "seuil critique" la valeur expérimentale de 18 kilobars mentionnée ci-dessus, on constate (figure 7) que l'épaisseur de l'écaille formée $(3+0,5 \mathrm{~mm}$ ) est comparable à celle mesurée aprés tir ( 3 à $4 \mathrm{~mm}$ ).

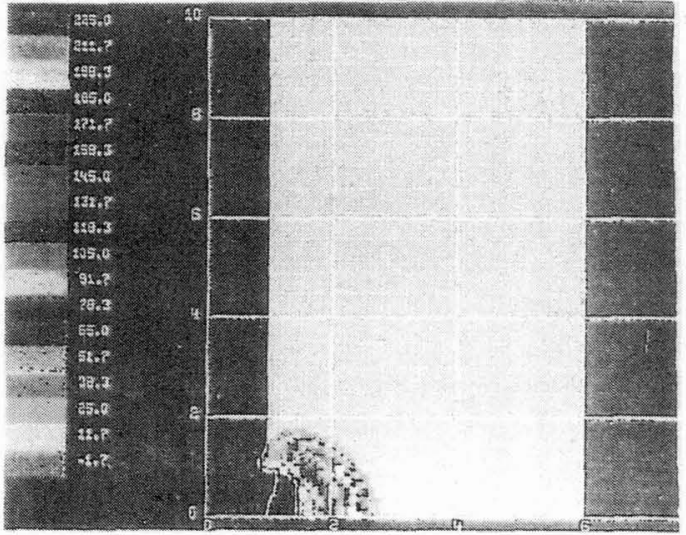

$2 \mu s$

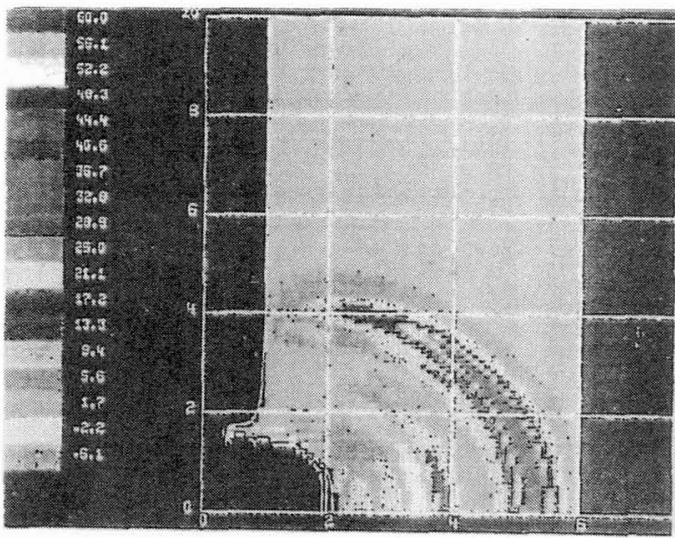

$7 \mu \mathrm{s}$

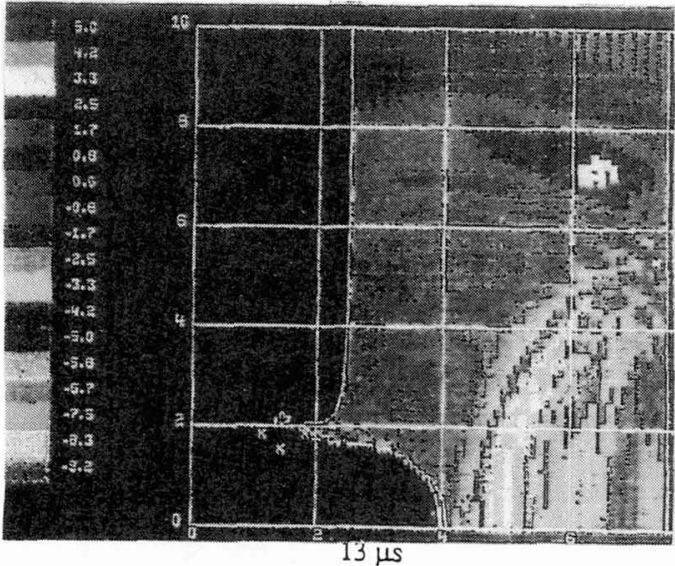

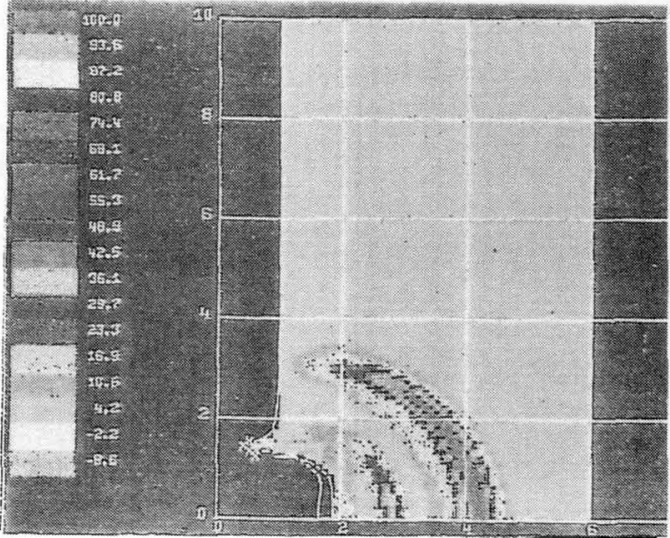

$5 \mu s$

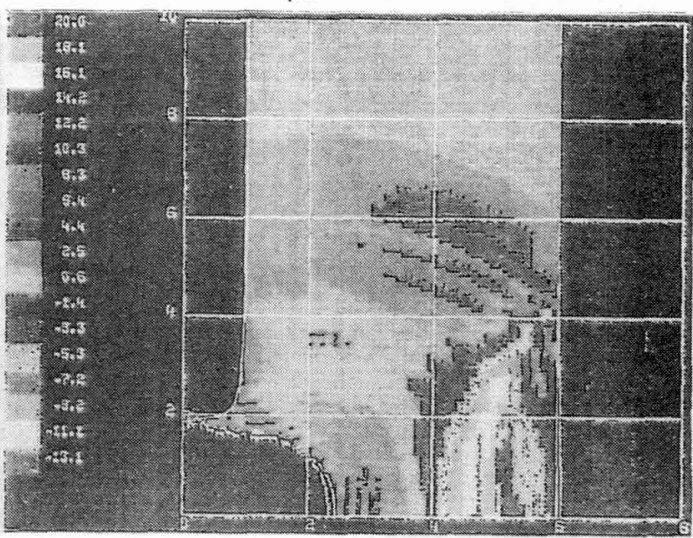

$10 \mu \mathrm{s}$

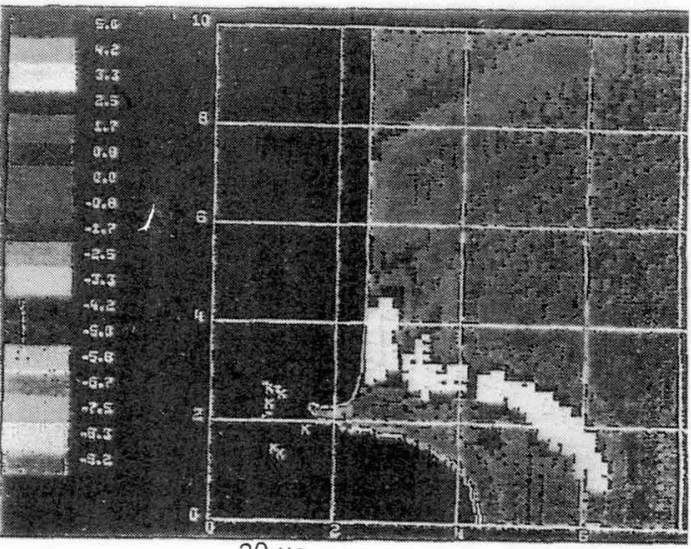

$20 \mu s$

Figure 6

Pression hydrostatique dans une cible en AU4G $(e=50 \mathrm{~mm})$ - Projectile de $2.15 \mathrm{~g}-\mathrm{v}=5400 \mathrm{~m} / \mathrm{s}$

(les pressions sont en kilobars - les temps sont comptés à partir de l'instant d'impact) 


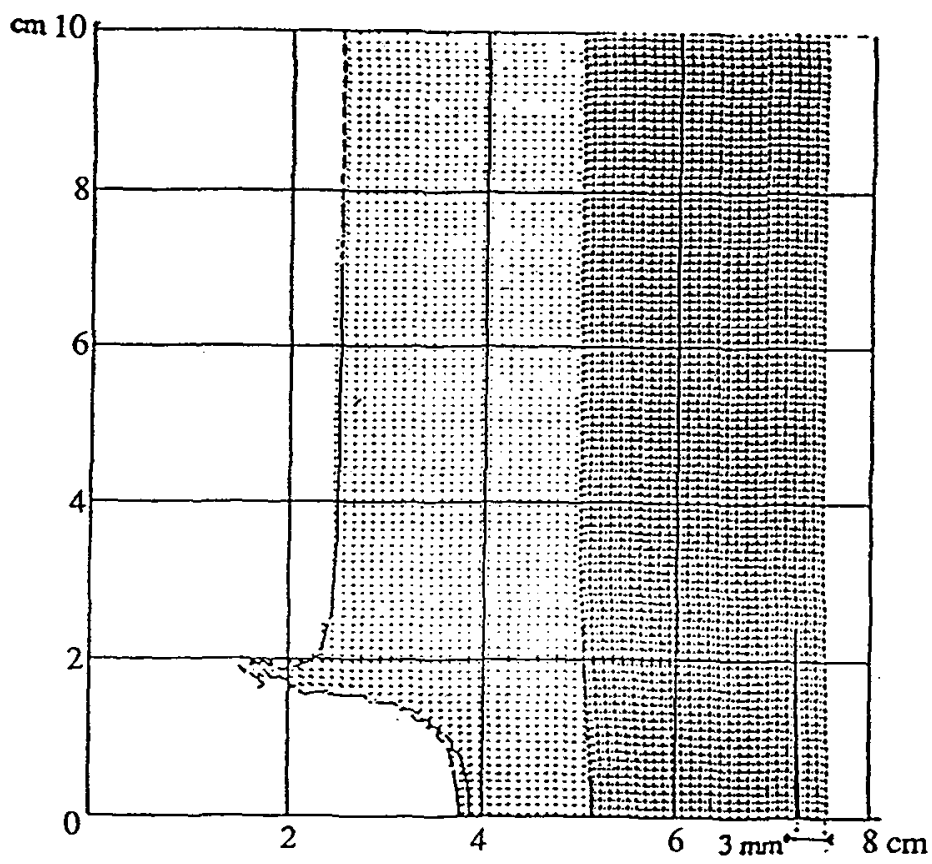

Figure 7 - Traitement de l'écaillage - aspect de la cible à $9.6 \mu \mathrm{s}$

Cible en AU4G $(e=50 \mathrm{~mm})$ - Projectile de $2,15 \mathrm{~g}-\mathrm{v}=5400 \mathrm{~m} / \mathrm{s}$ - calcul couplé Euler-Lagrange

Le tableau 2 rassemble les principaux points de comparaison expériences-calculs. Dans le cas où les effets sur la cible sont limités à la craterisation et à l'ouverture d'une écaille, les calculs permettent de bien restituer les dimensions du cratère et de l'écaille ; de plus, l'épaisseur critique de cible pour laquelle apparait une écaille est convenablement estimé à l'aide d'un critère de tension limite, aussi bien pour l'AU4G (seuil : 18 kilobars), que pour le TA6V (seuil : 45 kilobars).

Par contre, le code CEL ne possédant pas à ce jour de critère de fracturation, il ne peut pas simuler l'arrachement de l'écaille et, en particulier la diminution de profondeur du cratère lorsque l'énergie cinétique du projectile est suffisante pour provoquer l'ärrachement de l'écaille (phénomène classique déja observé par divers expérimentateurs $/ 6 /$ ) ; la comparaison expériencescalculs est alors moins favorable ('écart étant đe 20 à $30 \%$ ). A fortiori, la perforation complète des cibles minces ne peut être traitée.

Pour améliorer la restitution numérique des effets de limpact, un modèle de fracture et d'endommagement bidimensionnel est actuellement en cours dintroduction dans CEL; il comprend /8/:

- un critère de rupture ductile,qui consiste à calculer à chaque instant le rapport $P / Y$ de la pression hydrostatique à la contrainte d'écoulement effective et à comparer cette valeur à une "courbe critique de fracturation" fonction de la température et de la déformation plastique équivalente ;

- une loi de comportement de matériau fracturé basée, comme pour la plupart des modèles d'endommagement monodimensionnels, sur un traitement poreux : on définit une porosité $\alpha=\left(\rho_{f}-\rho\right) / \rho_{f}, \rho_{f}$ désignant la valeur de la densité atteinte à l'instant où le critère $\mathrm{P} / \mathrm{Y}$ est violé ; le comportement ultérieur du matériau est décrit à l'aide :

- d'un "module équivalent" $\mathrm{Eeq}=\mathrm{E}_{0} \mathrm{~g}(\alpha)$;

- d'une "courbe de traction plastique équivalente" $\sigma_{e q}=\sigma_{0}(\varepsilon, \varepsilon, \mathrm{T}) \mathrm{g}(\alpha)$, où $\mathrm{E}_{0}$ est le module d'Young initial, et $\sigma_{0}(\varepsilon, \varepsilon, T)$ la loi de comportement du matériau "vierge" (non endommagé).

Avec des modèles de ce type, on peut simuler aussi bien les fracturations au voisinage du cratère (sans doute dues à des cisaillements) que la formation et l'arrachement de l'écaille (pour lesquels le critère de nupture est plutôt duu type "contrainte principale maximale"), ce qui permet en particulier d'évaluer des seuils de perforation. 
Tableau 2- Comparaison expériences-calculs

\begin{tabular}{|c|c|c|c|c|c|c|c|}
\hline \multirow[t]{2}{*}{$\begin{array}{c}\text { Epaisseur } \\
\text { de cible (mm) }\end{array}$} & \multicolumn{2}{|c|}{$\begin{array}{c}\text { Cratère } \\
\text { diamètre (mm) }\end{array}$} & \multicolumn{2}{|c|}{$\begin{array}{c}\text { Cratère } \\
\text { profondeur }(\mathrm{mm})\end{array}$} & \multicolumn{2}{|c|}{$\begin{array}{c}\text { Epaisseur } \\
\text { d'écaille (mm) }\end{array}$} & \multirow{2}{*}{$\begin{array}{c}\begin{array}{c}\text { Tension max. } \\
\text { dans la cible (kbar) }\end{array} \\
\text { calc. }\end{array}$} \\
\hline & exp. & calc. & $\exp$ & calc. & exp. & calc. & \\
\hline \multicolumn{7}{|c|}{ Projectile $2,15 \mathrm{~g}-\nu=5400 \mathrm{~m} / \mathrm{s}-$ Cible en $A U 4 \mathrm{G}$} & Tens. lim.=18kbars \\
\hline $\begin{array}{l}50 \\
45 \\
40 \\
35\end{array}$ & $\begin{array}{l}42 \\
41 \\
42 \\
42\end{array}$ & $\begin{array}{l}40 \\
40 \\
40 \\
40\end{array}$ & $\begin{array}{r}20,5 \\
17,5 \\
-19 \\
\text { Perfo. }\end{array}$ & $\begin{array}{l}20,4 \\
20,7 \\
21,5 \\
22,4\end{array}$ & $\begin{array}{c}\text { seuil écaillage } \\
3 \text { à } 4 \\
-5 \\
-\end{array}$ & $\begin{array}{c}3,5 \\
3,5 \\
4 \\
4\end{array}$ & $\begin{array}{c}19 \\
23 \\
23,5 \\
25\end{array}$ \\
\hline \multicolumn{7}{|c|}{ Projectile $2,15 \mathrm{~g}-\mathrm{v}=5400 \mathrm{~m} / \mathrm{s}-$ Cible en TA6V } & Tens. $\lim .=45 \mathrm{kbars}$ \\
\hline $\begin{array}{l}45 \\
35\end{array}$ & $\begin{array}{l}37 \\
37\end{array}$ & $\begin{array}{l}38 \\
38\end{array}$ & $\begin{array}{l}12 \\
12\end{array}$ & $\begin{array}{l}10,1 \\
10,4\end{array}$ & $\begin{array}{l}\text { seuil écaillage } \\
2 \text { à } 4\end{array}$ & $\dot{2}$ & $\begin{array}{l}42 \\
55\end{array}$ \\
\hline \multicolumn{7}{|c|}{ Projectile $0,16 \mathrm{~g}-v=7200 \mathrm{~m} / \mathrm{s}-$ Cible en $A U 4 G$} & Tens. lim $=18 \mathrm{kbars}$ \\
\hline $\begin{array}{l}25 \\
20 \\
15\end{array}$ & $\begin{array}{c}17 \\
17 \\
?\end{array}$ & $\begin{array}{c}17 \\
19 \\
17.7\end{array}$ & $\begin{array}{l}9 \text { à } 10 \\
9 \\
\text { Perfo. }\end{array}$ & $\begin{array}{c}9,8 \\
11 \\
10,4\end{array}$ & $\begin{array}{c}\text { seuil écaillage } \\
-2 \\
-2\end{array}$ & $\begin{array}{l}2 \\
2 \\
2\end{array}$ & $\begin{array}{c}20 \\
50 \\
100\end{array}$ \\
\hline
\end{tabular}

\section{Référencers}

III H.C. PUJOLS - Lanceur hypervitesse à explosif - Communication pour la 40ème rencontre de l'Aeroballistic Range Association - (Paris - 25-28 Septembre 1989).

121 D.J. STEINBERG - S.G. COCHRAN - M.W. GUINAN - A constitutive model applicable at high strain rate- Journal of applied physics - 51 (3) - Mars 1980.

131 G.I. TAYLOR - The use of flat-ended projectiles for determining dynamic yield stress - Proc. R. Soc A194 - pages 289-299 - 1948.

141 M.L. WILKINS - M.W. GUINAN - Impact of cylinders on a rigid boundary - Journal of applied physics - 44 - pages 1200-1206- 1973.

15I W.H. GUST - High impact deformation of metal cylinders at elevated temperatures - Journal of applied physics - 53 (5) - pages 3566-3575 - 1982.

161 A.E. WILLIAMS - M.A. PERSECHINO - The effect of projectile properties on target cratering - Int. Journal of impact engineering - Vol. 5 - pages 709-728 - 1987.

I7) M. LACOMME - A. FROGER - J.P. ANSART - R. DORMEVAL - Endommagement sous choc d'un alliage d'aluminium AU4G - Communication présentée au DYMAT 88 - Journal de Physique C3 - 49 (9) - Septembre 1988.

181 M.A. NASH - I.G. CULLIS - Numerical modelling of fracture - a model for ductile fracture in triaxial states of stress Proc. 3rd conf. on the mechanical properties at high rates of strain (Oxford, 9-12 Avril 1984) - pages 307-314 - 1984. 